\title{
The American Diabetes Association's new standards for diabetes care
}

\author{
PAUL GRANT
}

\section{Introduction}

Diabetes care remains difficult in the sense that it can be poorly coordinated, overly clinician-centred, non-standardised, lacking in the right sort of evidence base and isn't integrated from a service user's perspective. 1,2 Do you agree? Do you run a good diabetes service? Have we successfully implemented ongoing patient self-management and education programmes and do our multidisciplinary teams offer the right type of support? If you were diagnosed with diabetes tomorrow, what type of care would you expect - nay demand?

\section{The American Diabetes Association approach}

Looking across the Atlantic, it is very interesting to read the latest position and scientific statements from the American Diabetes Association (ADA) in terms of their 'Standards of medical care in diabetes'. ${ }^{3}$ They state that the goal of the standards is to ensure that 'clinicians, health plans and policy makers can continue to rely on them as the most authoritative and current guidelines for diabetes care' and they include key clinical practice recommendations, using grade A or B evidence from up-to-date research. See how many of the following you agree with - or have already put into practice.

\section{Strategies for improving care}

- A patient-centred communication style.

- Timely treatment decisions, supported by evidence-based guidelines.

- Care should be aligned with the chronic care model (to ensure proactive interactions between a prepared proactive team and an informed activated patient).

- Care systems should support team-based care, community involvement, patient registries and decision support tools to meet patient needs.

2. Classification and diagnosis of diabetes (pre-diabetes)

- Testing to assess future risk of diabetes in an asymptomatic individual should be considered in those who are overweight (body mass index $(\mathrm{BMI})>25 \mathrm{~kg} / \mathrm{m}^{2}$ ) and have one or more risk factors for diabetes.

\section{OCDEM, Churchill Hospital, Oxford, UK}

Address for correspondence: Paul Grant

Consultant Diabetologist, OCDEM, Churchill Hospital, Oxford, UK

E-mail: Drpaul.grant@doctors.org.uk

Br J Diabetes 2016;16:99-100

http://dx.doi.org/10.15277/bjd.2016.086
- For all patients, testing should begin at 45 years of age and, if normal, carried out at three-yearly intervals. Fasting plasma glucose, two-hour oral glucose tolerance test and $\mathrm{HbA}_{1 \mathrm{c}}$ are all equally appropriate.

- Testing should be considered in children who are obese and have two or more risk factors for diabetes.

\section{Foundations of care and comprehensive medical} evaluation

- All people with diabetes should participate in diabetes selfmanagement education and support.

- For people with type 1 diabetes or those with type 2 diabetes who are prescribed a flexible insulin therapy programme, education on how to use carbohydrate counting or estimation to determine mealtime insulin dosing can improve glycaemic control.

- Adults with diabetes should be advised to perform at least 150 min/week of moderate intensity aerobic physical activity (50-70\% of maximum heart rate), spread over at least three days/week with no more than two consecutive days without exercise.

- The patient's psychological and social situation should be addressed in the medical management of diabetes. Routinely screen for psychosocial problems such as depression, diabetesrelated distress, anxiety, eating disorders and cognitive impairment.

4. Prevention or delay of type 2 diabetes

- Patients with pre-diabetes should be referred to an intensive diet and physical activity behavioural counselling programme, targeting a loss of $7 \%$ body weight, and should increase their moderate-intensity physical activity to at least 150 minutes per week.

- Metformin therapy for prevention of type 2 diabetes should be considered in those with pre-diabetes, especially in those with $\mathrm{BMI}>35 \mathrm{~kg} / \mathrm{m}^{2}$, those aged 60 years or over and women with prior gestational diabetes mellitus.

- Technology-assisted tools including internet-based social networks, distance learning, DVD-based content and mobile applications can be useful elements of effective lifestyle modification to prevent diabetes.

5. Glycaemic targets

- A reasonable $\mathrm{HbA}_{1 \mathrm{c}}$ goal for many non-pregnant adults is $7 \%$ (53 mmol/mol).

- Most patients on intensive insulin regimens (multiple-dose 
insulin or insulin pump therapy) should consider self-monitoring of blood glucose (SMBG) prior to meals and snacks, occasionally post-prandially, at bedtime, prior to exercise, when they suspect low blood glucose, after treating low blood glucose until they are normoglycaemic and prior to critical tasks such as driving.

- When used properly, continuous glucose monitoring (CGM) in conjunction with intensive insulin regimens is a useful tool to lower $\mathrm{HbA}_{1 c}$ in selected adults (aged 25 years) with type 1 diabetes.

- Although the evidence for $\mathrm{HbA}_{1 c}$ lowering is less strong in children, teens and younger adults, CGM may be helpful in these groups. Success correlates with adherence to ongoing use of the device.

- CGM may be a supplemental tool to SMBG in those with hypoglycaemia unawareness and/or frequent hypoglycaemic episodes.

\section{Obesity management}

- Bariatric surgery may be considered for adults with BMI 35 $\mathrm{kg} / \mathrm{m}^{2}$ and type 2 diabetes, especially if diabetes or associated co-morbidities are difficult to control with lifestyle and pharmacological therapy.

\section{Approaches to glycaemic control}

- Type 1 diabetes: most people with type 1 diabetes should be treated with multiple-dose insulin injections (three to four injections per day of basal and prandial insulin) or continuous subcutaneous insulin infusion. Consider educating individuals with type 1 diabetes on matching prandial insulin dose to carbohydrate intake, pre-meal blood glucose and anticipated activity.

- Type 2 diabetes: metformin, if not contraindicated and if tolerated, is the preferred initial pharmacological agent for type 2 diabetes. Consider initiating insulin therapy (with or without additional agents) in patients with newly diagnosed type 2 diabetes and markedly symptomatic and/or elevated blood glucose levels or $\mathrm{HbA}_{1 \mathrm{c}}$.

The standards go on to include guidance about the care of the older person with diabetes, screening and management of vascular complications, the management of children and adolescents and treatment of diabetes during pregnancy. I was most taken with section 13 , the management of diabetes in the hospital setting.

\section{Diabetes care in the hospital}

- Consider performing an $\mathrm{HbA}_{1 \mathrm{c}}$ on all patients with diabetes or hyperglycaemia admitted to the hospital if not performed in the prior 3 months.

- Insulin therapy should be initiated for treatment of persistent hyperglycaemia starting at a threshold of $180 \mathrm{mg} / \mathrm{dL}(10.0 \mathrm{mmol} / \mathrm{L})$. Once insulin therapy is started, a target glucose range of $140-180 \mathrm{mg} / \mathrm{dL}$ (7.8$10.0 \mathrm{mmol} / \mathrm{L})$ is recommended for the majority of critically ill patients and non-critically ill patients. More stringent goals such as $110-140 \mathrm{mg} / \mathrm{dL}(6.1-7.8$ $\mathrm{mmol} / \mathrm{L}$ ) may be appropriate for selected critically ill patients, as long as this can be achieved without significant hypoglycaemia.

- Intravenous insulin infusions should be administered using validated written or computerised protocols that allow for predefined adjustments in the insulin infusion rate based on glycaemic fluctuations and insulin dose.

- A basal plus bolus correction insulin regimen is the preferred treatment for non-critically ill patients with poor oral intake or those who are taking nothing by mouth. An insulin regimen with basal, nutritional and correction components is the preferred treatment for patients with good nutritional intake.

- The sole use of sliding scale insulin (variable rate intravenous insulin infusion) in the inpatient hospital setting is strongly discouraged.

- A hypoglycaemia management protocol should be adopted and implemented by each hospital or hospital system. A plan for preventing and treating hypoglycaemia should be established for each patient. Episodes of hypoglycaemia in the hospital should be documented in the medical record and tracked.

- The treatment regimen should be reviewed and changed if necessary to prevent further hypoglycaemia when a blood glucose value is $70 \mathrm{mg} / \mathrm{dL}$ (3.9 mmol/L).

- There should be a structured discharge plan tailored to the individual patient.

In summary, it is interesting how well the vast majority of the ADA standards chime with what we would expect to be good quality diabetes care in the UK. There is a focus on improving patient experience, education, identifying psychosocial issues and supporting self-management as well as thorough screening for complications, the evaluation of the efficacy of ongoing treatments and the recognised importance of up-titrating therapeutic regimes when we are not winning. The professional practice committee of the ADA has done a good job with their latest revisions and will find much in the way of European synchronicity of ideals.

\section{References}

1. Druce M. Addressing quality in endocrine care. Clin Endocrinol 2014;81:818-19. http://dx.doi.org/10.1111/cen.12544

2. Grant P. Should we be standardising diabetes care to reduce variation? Br J Diabetes Vasc Dis 2014; 14:30-4. http://dx.doi.org/10.15277/bjdvd.2014.006

3. ADA standards of medical care in diabetes 2016. Diabetes Care 2016; 39(S1). 